\title{
Evaluation of a Novel Absorbable Radiopaque Hydrogel in Patients Undergoing Image Guided Radiation Therapy for Borderline Resectable and Locally Advanced Pancreatic Adenocarcinoma
}

\author{
Avani Dholakia Rao, MD ${ }^{\mathrm{a}, 1}$, Eun Ji Shin, MD, PhD ${ }^{\mathrm{b}, 1}$, Jeffrey Meyer, MS, MDa, Elizabeth L. \\ Thompson, MD $^{c}$, Wei Fu, MS ${ }^{d}$, Chen Hu, MS, PhD ${ }^{d}$, Elliot K. Fishman, MD ${ }^{e}$, Matthew Weiss, \\ MD $^{f}$, Christopher Wolfgang, MD, PhD ${ }^{\dagger}$, Richard Burkhart, MD $^{f}$, Jin He, MD, PhD ${ }^{f}$, Tossapol

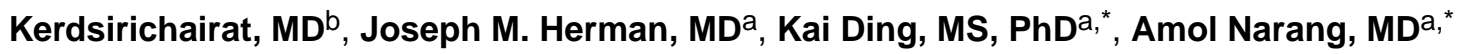 \\ aDepartment of Radiation Oncology and Molecular Radiation Sciences, The Sol Goldman \\ Pancreatic Cancer Research Center, Johns Hopkins School of Medicine, Baltimore, Maryland \\ bDepartment of Gastroenterology, The Sol Goldman Pancreatic Cancer Research Center, Johns \\ Hopkins School of Medicine, Baltimore, Maryland 'Department of Pathology, The Sol Goldman \\ Pancreatic Cancer Research Center, Johns Hopkins School of Medicine, Baltimore, Maryland \\ dDivision of Biostatistics and Bioinformatics, Sidney Kimmel Comprehensive Cancer Center, \\ Johns Hopkins School of Medicine, Baltimore, Maryland eDepartment of Radiology and \\ Radiological Sciences, Johns Hopkins School of Medicine, Baltimore, Maryland fDepartment of \\ Surgery, Johns Hopkins School of Medicine, Baltimore, Maryland
}

\section{Abstract}

\begin{abstract}
Purpose-We assessed the feasibility and safety of placing a radiopaque hydrogel in the pancreaticoduodenal groove via endoscopic ultrasound guidance in patients with borderline resectable/locally advanced pancreatic cancer (BR/LAPC).
\end{abstract}

\begin{abstract}
Methods and Materials-Hydrogel injections were done at time of fiducial placement to form blebs in the pancreaticoduodenal groove. Patients subsequently underwent simulation computed tomography (sim-CT) followed by hypofractionated stereotactic body radiotherapy (SBRT; 33 Gy in 5 fractions). Four to 8 weeks after SBRT, patients underwent CT re-evaluation for surgical candidacy and assessment of hydrogel location and size. Hydrogel placement was considered successful if identified in the pancreaticoduodenal groove on sim-CT scan. Stability was evaluated using equivalence testing analyses, with a null hypothesis of the presence of a $\geq 20 \%$ mean percentage change in volume and $x$ change in the median and mean interbleb surface distance with a $P$ value $<.05$ required to reject the null hypothesis and conclude equivalence. For patients undergoing pancreaticoduodenectomy, hydrogel sites were histologically examined for location and local inflammatory reactions.
\end{abstract}

\footnotetext{
"Corresponding authors: Kai Ding, MS, PhD and Amol Narang, MD; kding1@jhmi.edu and anarang2@jhmi.edu.

${ }^{1}$ A.D.R. and E.J.S. contributed equally to this work.
} 
Results-Hydrogel placement was successful in 6 of the 6 evaluable patients. The average changes in median and mean interbleb distances were $-0.43 \mathrm{~mm}$ and $-0.35 \mathrm{~mm}$, respectively, with $P<.05$. The average change in volume from sim-CT to post-SBRT CT was $-1.0 \%$, with $P<.05$. One patient experienced grade 3 nausea after fiducial/hydrogel placement, with no other adverse events to date.

Conclusions-These data demonstrate feasibility and safety of injecting a hydrogel marker in the pancreaticoduodenal groove in patients with BR/LAPC and set the stage for a follow-up clinical trial to place hydrogel as a spacer between the pancreatic tumor and dose-limiting, radiosensitive duodenum.

\section{Introduction}

For patients with borderline resectable and locally advanced pancreatic cancer (BR/LAPC), the main challenges to the delivery of dose-escalated radiation therapy (RT) include proximity of the radiosensitive duodenum adjacent to the head of the pancreas (HOP) and limitations in soft tissue contrast of on-board cone beam computed tomography, prohibiting clinicians from being able to visualize this boundary during treatment. ${ }^{1}$

Our group has previously reported the feasibility of placing a radiopaque hydrogel (TraceIT, Augmenix, Bedford, MA) between the HOP and duodenum to function as a marker and spacer in both in human cadaveric, ${ }^{2}$ porcine, ${ }^{3}$ and patient simulation ${ }^{4}$ studies, with additional support for safety in the porcine studies. ${ }^{3}$ TraceIT is a high-contrast hydrogel made of iodinated polyethylene glycol and water ( $\sim 90 \%$ water, $9.25 \%$ polyethylene glycol, and $0.75 \%$ iodine). The hydrogel paste creates a bleb of particles at the needle tip on injection. This bleb remains dimensionally stable for 3 months and is fully absorbed after 7 months. The hydrogel has a specific gravity of 1.02 , and in the treatment planning process can be treated as water.

We report the experience of a clinical trial (NCT03307564) to assess the feasibility and safety of placing hydrogel between the HOP-duodenum interface via endoscopic ultrasound (EUS) guidance for the first time in patients with BR/LAPC. As this is the first in-human experience to attempt to use the hydrogel tissue marker to localize this important boundary, hydrogel injection was restricted to a small volume ( $<1 \mathrm{~mL}$ per injection).

\section{Methods and Materials}

\section{Study design}

After approval from the Johns Hopkins Institutional Review Boards Institutional Review Board, patients were enrolled on this prospective, single institution, single arm pilot study (NCT03307564) to evaluate the feasibility of injecting TraceIT Tissue Marker to mark the interface between the pancreas and duodenum. Six patients with pathologically confirmed BR/LAPC pancreatic adenocarcinoma delineable $>1 \mathrm{~mm}$ from the duodenal wall planned for stereotactic body radiotherapy (SBRT) based on multidisciplinary consensus were enrolled.

Enrolled subjects were scheduled for TraceIT placement at the time of fiducial procedure. Fiducials were implanted as previously described using an EUS-guided transduodenal 
approach. ${ }^{5}$ After fiducial placement, the pancreaticoduodenal groove was identified using a linear echoendoscope. The hydrogel was diluted with saline (3:1, 2:1, 1:1, TraceIT:Saline) to reduce viscosity for injection, with dilution concentration determined at time of injection attempt based on perceived resistance to injection at the HOP-duodenum interface. A 22gauge EUS needle was used to inject a total of 3 to 4 hydrogel blebs, formed by $1 \mathrm{~mL}$ of TraceIT (or dilute TraceIT solution) along the HOP-duodenum interface. After fiducial and hydrogel placement, patients underwent computed tomography simulation (median 1 day postfiducial and hydrogel placement), followed by hypofractionated SBRT (33 Gy in 5 fractions) within 1 month from simulation as previously described. ${ }^{6,7}$ After SBRT, patients proceeded with standard of care follow-up, which included CT scan at 4 to 8 weeks after SBRT to determine resectability. Patients were assessed for side effects from hydrogel placement at the time of the placement procedure, simulation, and surgical re-evaluation visit.

If a patient on study proceeded to pancreaticoduodenectomy, the hydrogel injection sites between pancreas and duodenum were examined in the surgical specimen for implant location and local inflammatory reactions, scored on a scale of 1 (none or minimal signs of mucosal damage) to 3 (severe damage) using methods outlined by Verma et al. ${ }^{8}$ Tissues were fixed in formalin, he matoxylin and eosin stained, and sectioned for analysis.

\section{Statistical analysis}

Data were collected using Velocity software (Varian Medical Systems, Palo Alto, CA) and analyzed in $\mathrm{R}$ version 3.5.3 ( $\mathrm{R}$ Core Team, 2019). The placement procedure was determined successful if the hydrogel was identified between the region of the pancreatic tumor and the duodenum on axial cross-sectional planning CT scan. To assess stability, individual bleb volume and the surface distances of points on one bleb surface to the nearest point on the second bleb were calculated. The median and mean of the surface distances between any pair of blebs were used allowing for consistent assessment of spatial relationship among blebs on simulation and post-SBRT CT. Stability was evaluated using equivalence testing analyses, with a null hypothesis of the presence of a $20 \%$ mean percentage change in volume and $\geq 2 \mathrm{~mm}$ change in the median and mean interbleb distance with a $P$ value $<.05$ required to reject the null hypothesis and conclude equivalence. Equivalence testing thresholds were selected to reflect the estimated uncertainty in measurement of scans that were performed using a 2-mm slice thickness for blebs that were generated using sequential $1-\mathrm{mL}$ injections.

\section{Results}

\section{Hydrogel placement procedure}

All patients $(n=6)$ underwent successful hydrogel placement (Table 1) with appropriate position confirmed on cross-sectional CT as shown in a representative case in Figure 1. Only one adverse event was deemed potentially attributable to the EUS-guided hydrogel placement. Patient 2 experienced grade 3 nausea after recovery from anesthesia; however, in addition to hydrogel placement, the patient had undergone anesthesia and several research biopsies, which may have contributed to these symptoms. 


\section{Hydrogel stability}

The median and mean interbleb distances were measured on SBRT simulation CT and compared with the interbleb distances at 4 to 8 weeks post-SBRT CT are shown in Table 2 . The average of the changes (post-minus pre-SBRT) in median and mean interbleb distances were $-0.43 \mathrm{~mm}$ (standard error, $0.95 \mathrm{~mm}$ ) and $-0.35 \mathrm{~mm}$ (standard error, $0.95 \mathrm{~mm}$ ), respectively, with $P<.05$ supporting equivalence of distance measurements with equivalence margin of $2 \mathrm{~mm}$. The average of the changes in hydrogel bleb volume from simulation CT to post-SBRT CT was $-1.0 \%$ (range, $-16.3 \%$ to $17.1 \%$ ), with $P<.05$ supporting the equivalence of the volumes across the cohort (Table 3).

\section{Histopathologic assessment}

Five patients underwent subsequent resection of their pancreatic primary, with 4 patients undergoing pancreaticoduodenectomy and 1 patient undergoing distal pancreatectomy (Table 1). In the 4 patients undergoing pancreaticoduodenectomy allowing for resection of hydrogel blebs in the surgical specimen, placement in the pancreaticoduodenal groove was confirmed, but hydrogel was also noted in regions within the duodenal submucosa with giant cell and granulomatous reaction as shown in Figure 2. There was no evidence of microscopic (score of $1=$ no/minimal signs of damage) or gross duodenal ulceration in the evaluable patients.

\section{Discussion}

We demonstrate the feasibility and safety of placing hydrogel in the pancreaticoduodenal groove in this first in-human trial exploring this technique of hydrogel injection via EUS guidance in patients with BR/LAPC. We further demonstrate the stability of the relationship of the hydrogel blebs and bleb volume.

Existing preliminary data support optimism for the possibility of improving outcomes for patients with unresectable pancreatic cancer with dose-escalated RT. ${ }^{9-11}$ Having developed this technique of hydrogel placement in the pancreaticoduodenal groove presented here, we are now enrolling patients with BR/LAPC on a study to inject a larger volume of hydrogel with the ultimate goal of serving as a spacer to reduce risk of duodenal toxicity and provide the opportunity for dose-escalated radiation therapy for patients with BR/LAPC.

\section{Acknowledgments}

Sources of support: Augmenix (Bedford, MA) provided a research grant to fund the clinical trial reported in this article; however, data were collected, analyzed, and reported independently from the sponsor. Research reported in this publication was supported in part by the National Cancer Institute of the National Institutes of Health under Award Number R37CA229417. The content is solely the responsibility of the authors and does not necessarily represent the official views of the National Institutes of Health.

Disclosures: Dr Rao reports grants from Augmenix (related to the submitted work), during the conduct of the study. Dr Shin reports grants from National Cancer Institute and Augmenix (related to the submitted work), during the conduct of the study. Dr Meyer reports grants from Augmenix (related to the submitted work), during the conduct of the study; other from UpToDate, Inc, outside the submitted work. Dr Hu reports grants from National Cancer Institute, grants from American College of Radiology, and personal fees from Merck \& Co, outside the submitted work. Dr Herman reports grants, personal fees, other from Augmenix, and personal fees from Boston Scientific, during the conduct of the study (related to the submitted work); personal fees from Bristol-Meyers Squibb, grants, personal fees, and other from Astra-Zeneca, personal fees and other from Medtronic, grants from Oncosil, and 
grants from Galera, outside the submitted work. Dr Ding reports grants from National Cancer Institute and Augmenix (related to the submitted work), during the conduct of the study. Dr Narang reports grants from National Cancer Institute and Augmenix (related to the submitted work), during the conduct of the study. Drs Thompson, Fishman, Weiss, Wolfgang, Burkhart, He, Kerdsirichariat, and Wei Fu have nothing to disclose.

\section{References}

1. Rudra S, Narang AK, Pawlik TM, et al. Evaluation of predictive variables in locally advanced pancreatic adenocarcinoma patients receiving definitive chemoradiation. Pract Radiat Oncol. 2012;2:77-85. [PubMed: 23585823]

2. Rao AD, Feng Z, Shin EJ, et al. Novel use of a biodegradable radiopaque hydrogel spacer as a technique to separate the head of the pancreas and duodenum in radiotherapy in pancreatic cancer. Int J Radiat Oncol Biol Phys. 2017;99:1111-1120. [PubMed: 28943075]

3. Rao AD, Shin EJ, Beck S, et al. Demonstration of safety and feasibility of hydrogel marking of the pancreas-duodenum interface for image-guided radiation therapy (IGRT) in a porcine model: Implications for IGRT for pancreatic cancer patients. Int J Radiat Oncol Biol Phys. 2018;101:640645. [PubMed: 29680252]

4. Feng Z, Rao AD, Cheng Z, et al. A dose prediction model for duodenum sparing with biodegradable hydrogel spacer in radiotherapy of pancreatic cancer. Int J Radiat Oncol Biol Phys. 2018;103:651659.

5. Khashab MA, Kim KJ, Tryggestad EJ, et al. Comparative analysis of traditional and coiled fiducials implanted during EUS for pancreatic cancer patients receiving stereotactic body radiation therapy. Gastrointest Endosc. 2012;76:962-971. [PubMed: 23078921]

6. Herman JM, Chang DT, Goodman KA, et al. Phase II multi-institutional trial evaluating gemcitabine and stereotactic body radiation therapy for locally advanced unresectable pancreatic adenocarcinoma. Cancer. 2015;121:1128-1137. [PubMed: 25538019]

7. Moningi S, Dholakia AS, Raman SP, et al. Stereotactic body radiation therapy for pancreatic cancer: single institutional experience. Ann Surg Onc. 2015;22:2352-2358.

8. Verma V, Lazenby AJ, Zheng D, et al. Dosimetric parameters correlate with duodenal histopathologic damage after stereotactic body radiotherapy for pancreatic cancer: Secondary analysis of a prospective clinical trial. Radiother Oncol. 2017;122:464-469. [PubMed: 28089484]

9. Krishnan S, Chadha AS, Suh Y, et al. Focal radiation therapy dose escalation improves overall survival in locally advanced pancreatic cancer patients receiving induction chemotherapy and consolidative chemoradiation. Int J Radiat Oncol Biol Phys. 2016; 94:755-765. [PubMed: 26972648]

10. Rudra S, Jiang N, Rosenberg SA, et al. Using adaptive magnetic resonance image-guided radiation therapy for treatment of inoperable pancreatic cancer. Cancer Med. 2019;8:2123-2132. [PubMed: 30932367]

11. Jiang N, Cao M, Lamb JM, et al. Outcomes utilizing MRI-guided and real-time adaptive pancreas stereotactic body radiotherapy (SBRT). Int J Radiat Oncol Biol Phys. 2017;99:S146. 

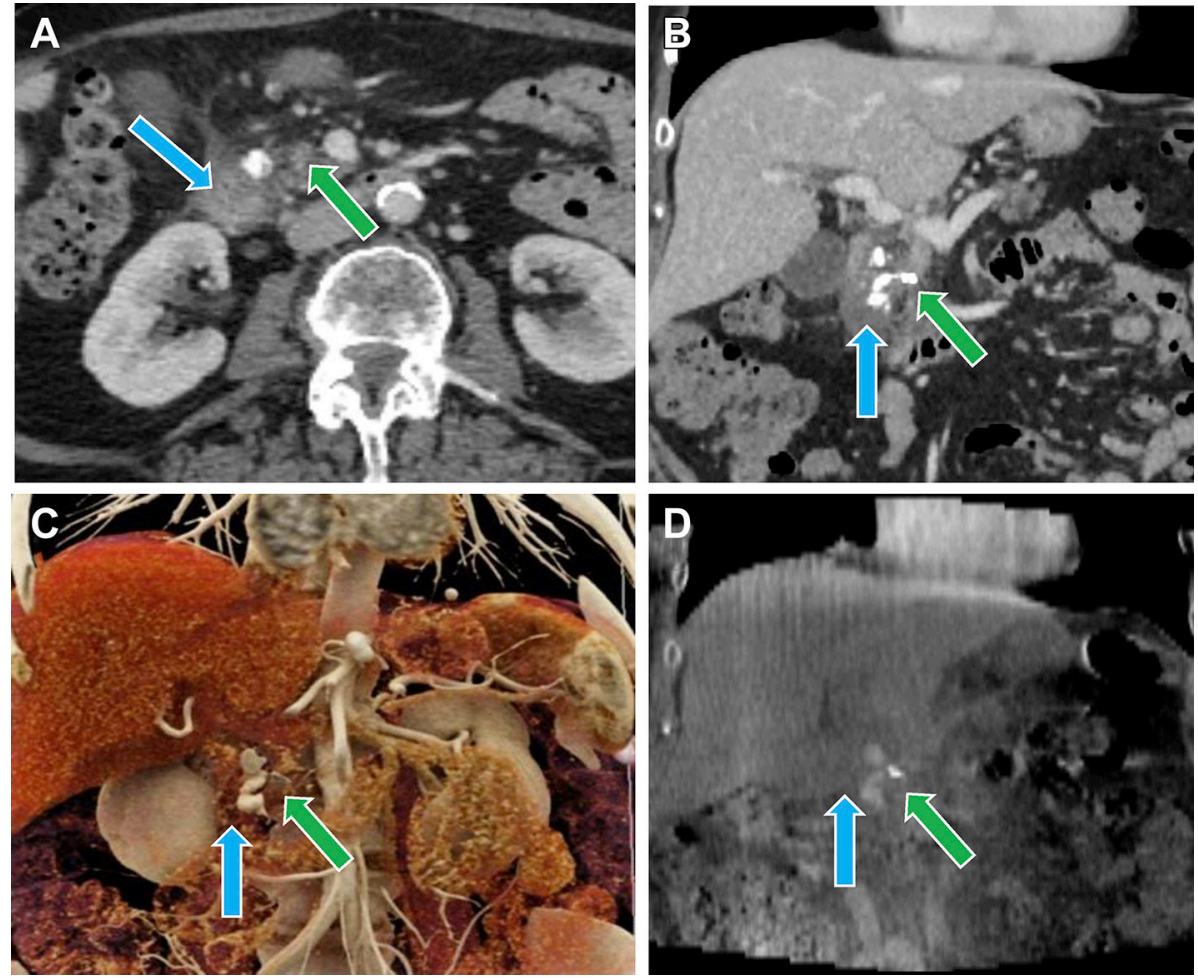

Figure 1.

Hydrogel placement confirmation on computed tomography and visibility on cone beam computed tomography. Hydrogel blebs between the pancreatic mass (green arrow) and duodenum (blue arrow) are clearly visible on coronal and axial computed tomographic views (A, B), 3-dimensional reconstruction (C), and cone beam computed tomography (D) in a representative case of patient 4. 

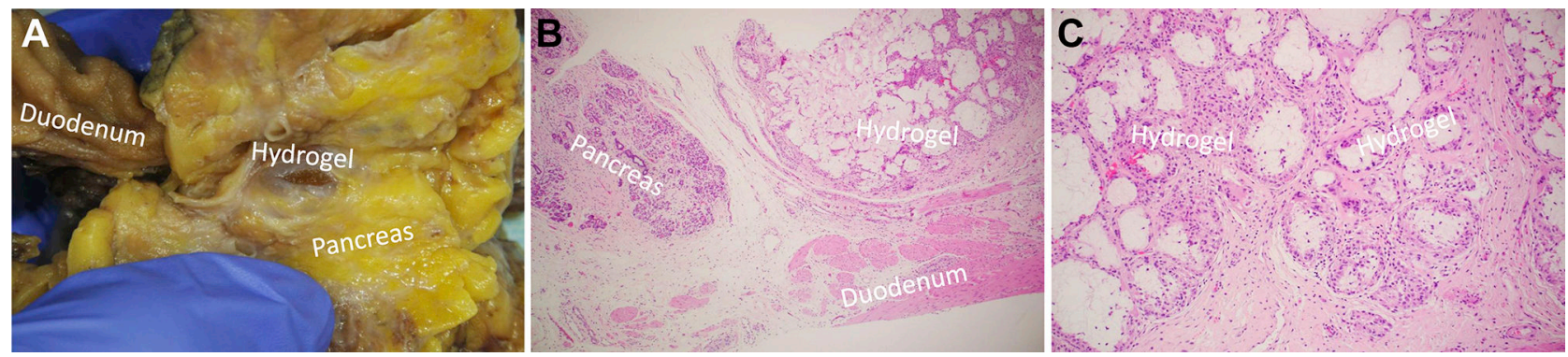

Figure 2.

Histopathologic analysis of hydrogel placement. Gross examination (A) of hydrogel placement in the pancreaticoduodenal groove. Histopathologic examination shows hydrogel placement in the pancreaticoduodenal groove (B) and regions within the duodenal submucosa with giant cell and granulomatous reaction without evidence of microscopic or gross ulceration $(C)$. Original magnification $(B, C) \times 20(2 \times 10)$. 


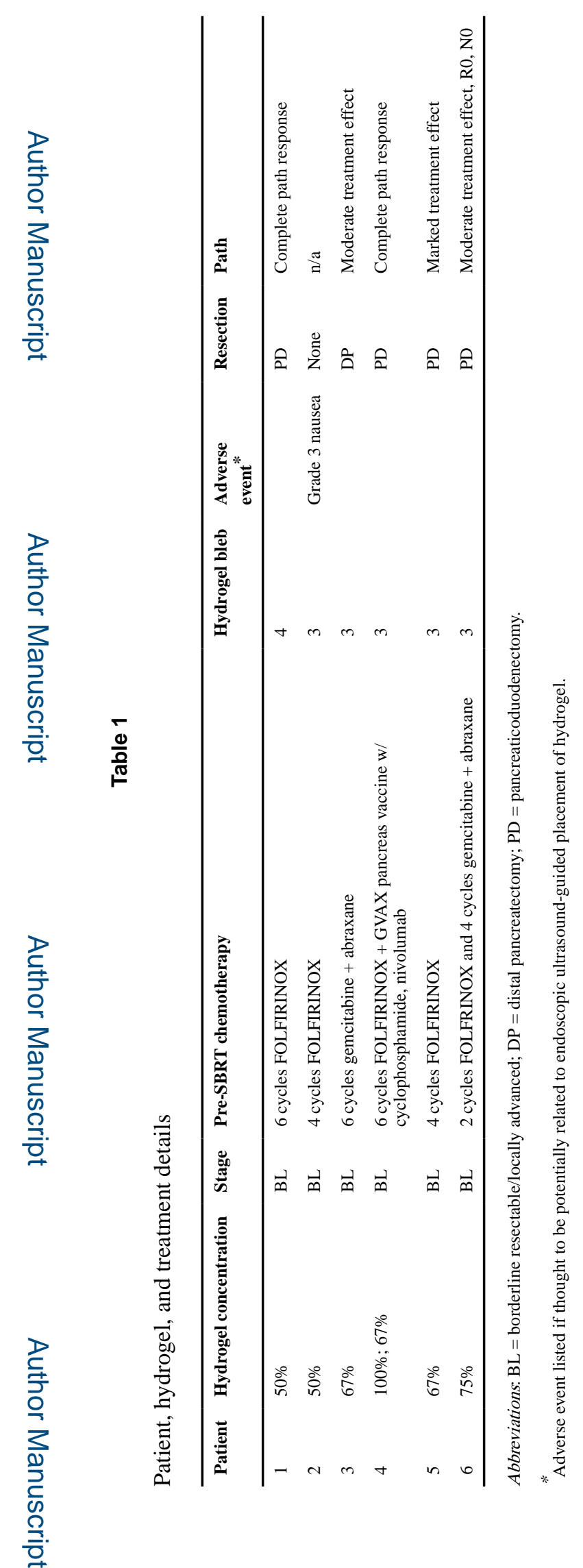

Pract Radiat Oncol. Author manuscript; available in PMC 2021 November 01. 


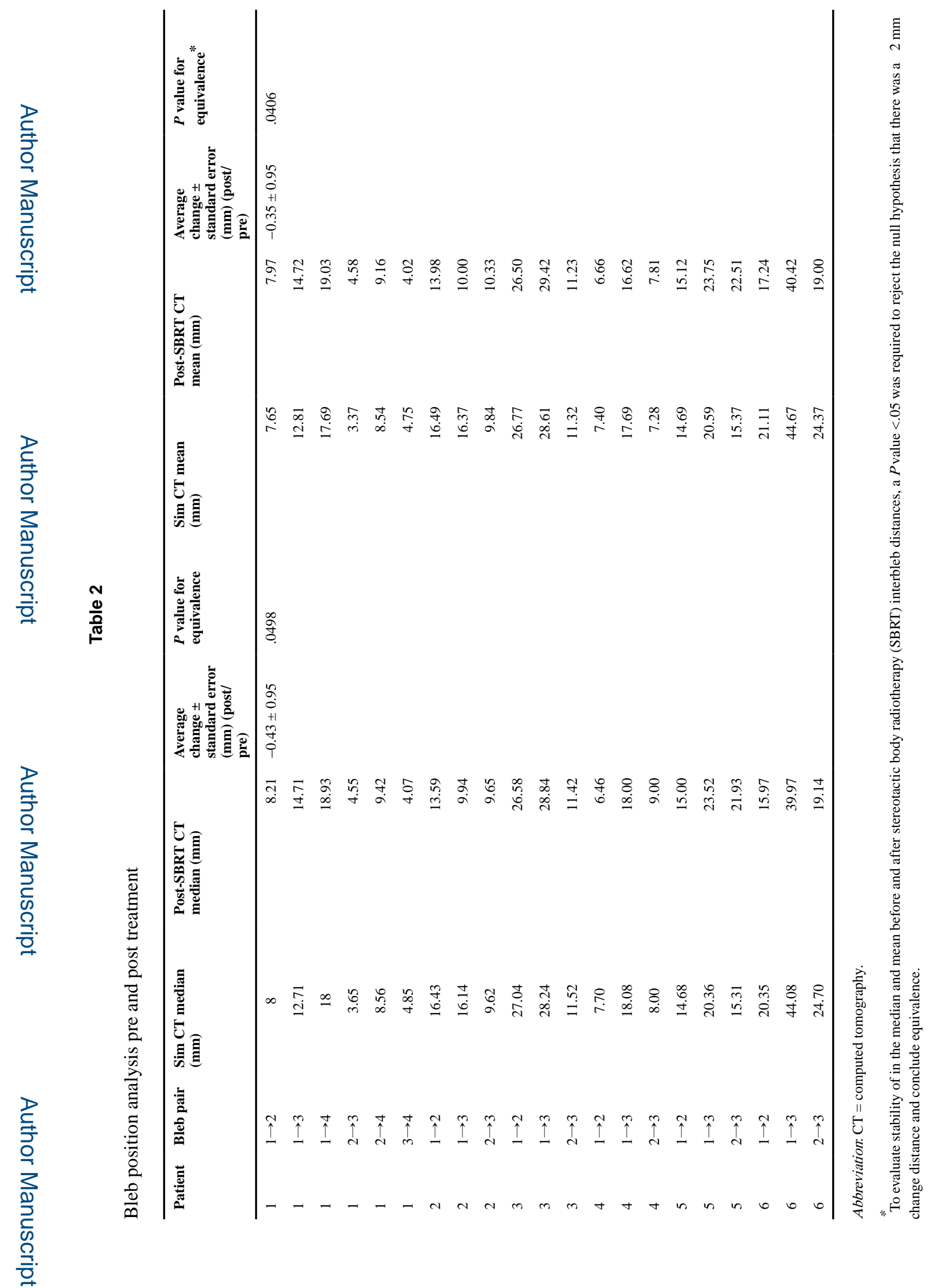

Pract Radiat Oncol. Author manuscript; available in PMC 2021 November 01. 


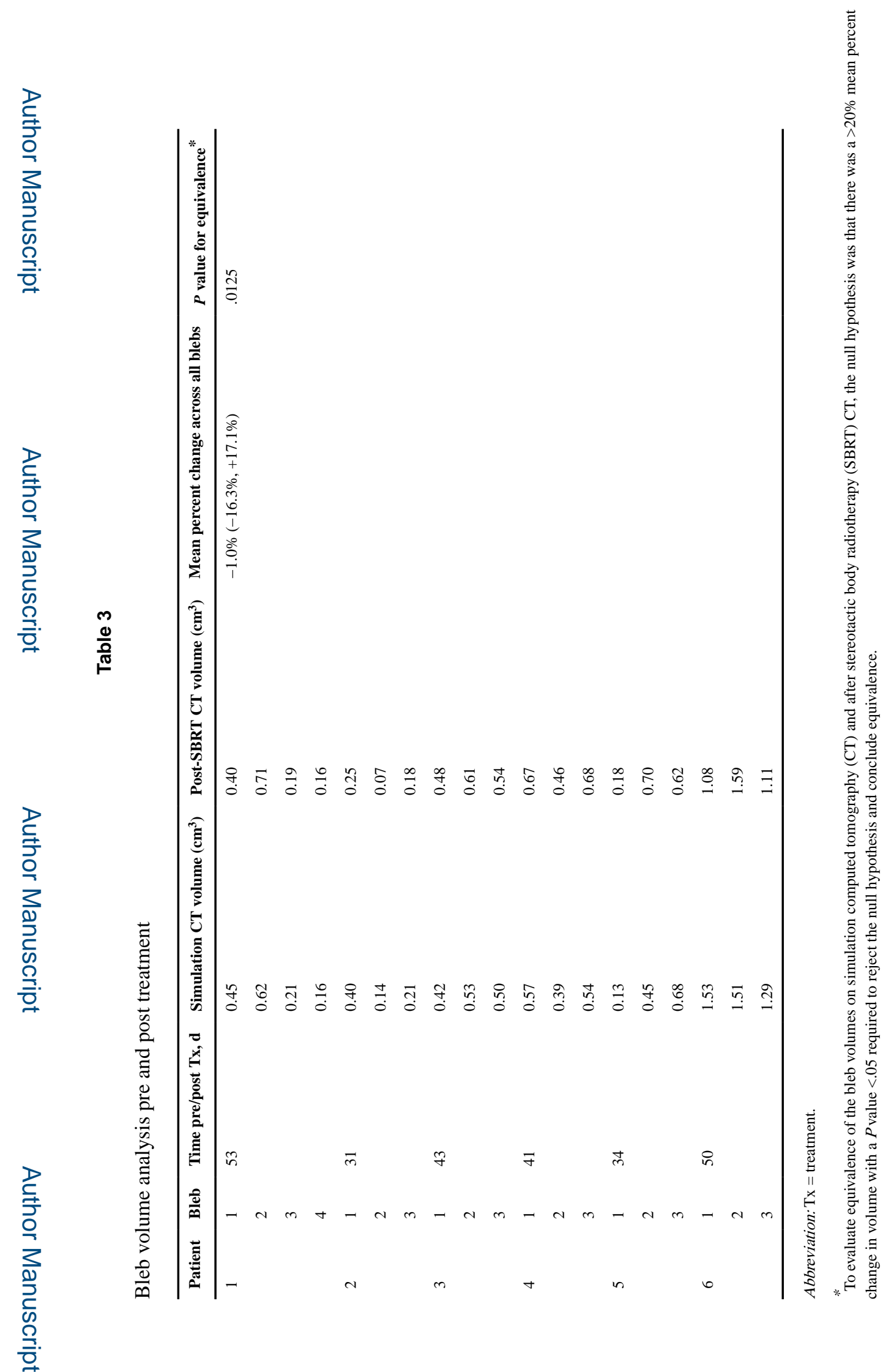

Pract Radiat Oncol. Author manuscript; available in PMC 2021 November 01. 